\title{
AUV NAVIGATION BASED ON MULTI-SENSOR FUSION FOR BREAKWATER OBSERVATION
}

\author{
Hayato Kondo \\ Faculty of Marine Technology \\ Tokyo University of Marine Science and Technology \\ 2-1-6, Etchujima, Koto, Tokyo, 135-8533, Japan \\ hkondo@e.kaiyodai.ac.jp
}

\author{
Toshihiro Maki, Tamaki Ura, Takashi Sakamaki \\ Underwater Technology Research Center \\ IIS, The University of Tokyo \\ 4-6-1, Komaba, Meguro, Tokyo, 153-8505, Japan \\ \{maki, ura, sakamaki\}@iis.u-tokyo.ac.jp
}

\begin{abstract}
Autonomous Underwater Vehicles (AUVs) are suitable for underwater observation, since they can swim freely without an umbilical cable. This paper proposes a robust navigation method for AUVs operating around artificial structures such as piers, quays and breakwaters. This method consists of a State Estimator and a Guidance System, enabling localization in the configuration map as well as following pre-given waypoints without any support systems, except its own sensors. The state estimator is based on a particle filter where the vehicle's state, horizontal position and heading, is stochastically updated in real time using multi-sensor data. This method was actually implemented to a testbed AUV "Tri-Dog 1" and a series of sea trials were carried out around breakwater caissons at Kamaishi bay in Japan. To verify the performance, mosaics of the breakwater are made from the observed images based on the estimated position and attitude of the AUV.
\end{abstract}

Keywords: Autonomous Underwater Vehicle, Navigation, Sensor fusion, Particle filter, Mosaic

\section{INTRODUCTION}

Condition surveys of artificial underwater structures such as caisson's surface, foot protection blocks and rock mound are very important for construction, maintenance and disaster-relief. Although divers and Remotely Operated Vehicles (ROVs) are playing an important role on this work, they have some inherent shortcomings. Divers work is a dangerous condition and can not reach great depth. ROVs require an umbilical cable that restricts their range and mobility.

AUVs can operate without an umbilical cable and the demand for AUVs that can undertake such missions is increasing. The most challenging hurdle to overcome is localization. GPS cannot be used underwater, so acoustic positioning systems such as Long Base Line (LBL), Short Base Line (SBL) and Super Short Base Line (SSBL) are often used. However, acoustic positioning systems require external support systems and are not suitable for operations around structures, where problems of multipath and acoustic shadows are unavoidable. At the same time, there is no comprehensively applicable solution to error accumulation when dead reckoning using DVL (Doppler Velocity Log) and INS (Inertial Navigation System). Structure based localization methods are proposed for some applications $[1,2]$. Although they need no external help like acoustic transponders, they are not robust against sensory noise since they are single-sensor systems. An oceangoing AUV must cope with various types of unexpected conditions under which single-sensor systems easily fail. The need of multi-sensor fusion is highlighted and some attempts are

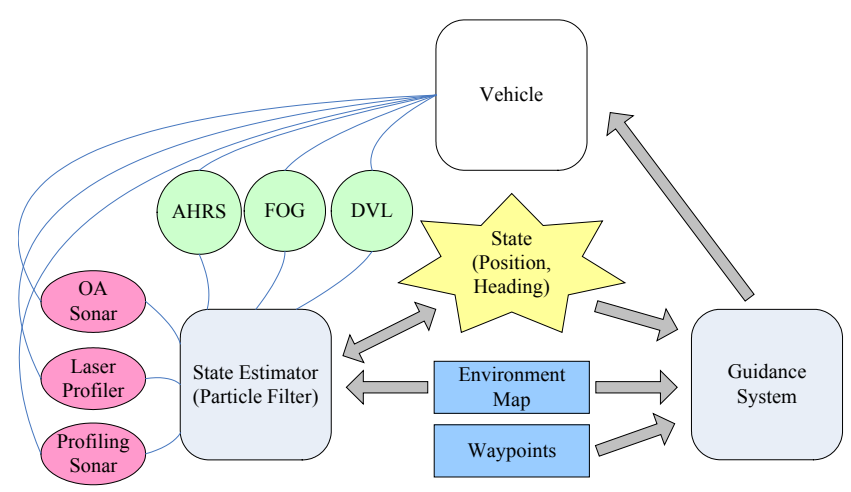

Fig. 1. Fusion based navigation method.

made with limited success [3 - 5].

We proposed a fusion based localization scheme to develop a practical navigation method for an AUV working around artificial structures, and the performance of the method was verified through tank trials [6], using a testbed AUV "Tri-Dog 1" [7]. The method utilizes probabilistic approach called "particle filter" $[8,9]$ and enables an AUV to localize itself in real time and follow pre-given waypoints without any external help, referring to sensory data and the map of the environment where the AUV is deployed.

To verify practical availability of the method, sea trials were carried out around breakwater caissons of Kamaishi bay, Iwate Prefecture in Japan. The AUV "Tri-Dog 1" carried out practical observations. This paper reports the results of the sea trials to evaluate the practical performance of the method. 


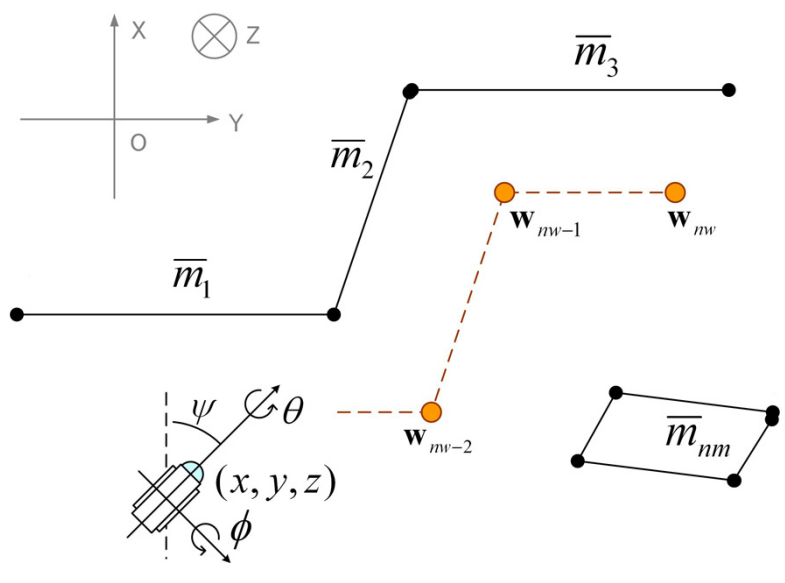

Fig. 2. Environment map M and waypoints W.

\section{NAVIGATION METHOD}

The method consists of two parts, the State Estimator and the Guidance System as shown in Fig. 1. The State Estimator estimates the state of the AUV;

$$
\mathbf{x}=\{\mathbf{r}, \psi\}
$$

using onboard sensors and environmental map $M$, where $\mathbf{r}$ and $\psi$ represent the horizontal position $[x, y]^{\mathrm{T}}$ and heading angle respectively. There is no need to estimate roll angle $\theta$, pitch angle $\phi$ and depth $z$ as they can be precisely measured by onboard sensors. The Guidance System outputs the control reference $R$ to follow the observation path $W$, using the estimated state $\mathbf{x}$ and the map $M$.

The map $M$ is given as a set of segments $\left\{\bar{m}_{1}, \bar{m}_{2}, \cdots, \bar{m}_{n m}\right\}$ and the observation path $W$ is defined as a set of waypoints $\left\{\mathbf{w}_{1}, \mathbf{w}_{2}, \cdots, \mathbf{w}_{n w}\right\}$ as shown in Fig. 2. The control reference $R$ consists of surge velocity $u$, sway velocity $v$, heading angular velocity $\omega$ and depth $z$.

\subsection{State Estimator}

Localization of an AUV should be treated as a stochastic state estimation problem, for every sensor measurement has random error. The particle filter is a powerful method that determines the probability density function of vehicle's state $p(\mathbf{x})$, described by $N$ samples

$$
S=\left\{\mathbf{s}^{1}, \mathbf{s}^{2}, \cdots, \mathbf{s}^{N}\right\}
$$

which are assumed to be randomly drawn from $p(\mathbf{x})$.

The state at the time $t$ is described by an average of the samples. The samples are updated recursively for each successive time step through the prediction phase and the observation phase.

\subsubsection{Prediction Phase}

During the prediction phase, the sample set $S_{t-1}$ is moved to $S^{\prime}{ }_{t}$ in accordance with an action $\mathbf{a}_{t-1}$ of the vehicle measured during the time period $[t-1, t]$. Each

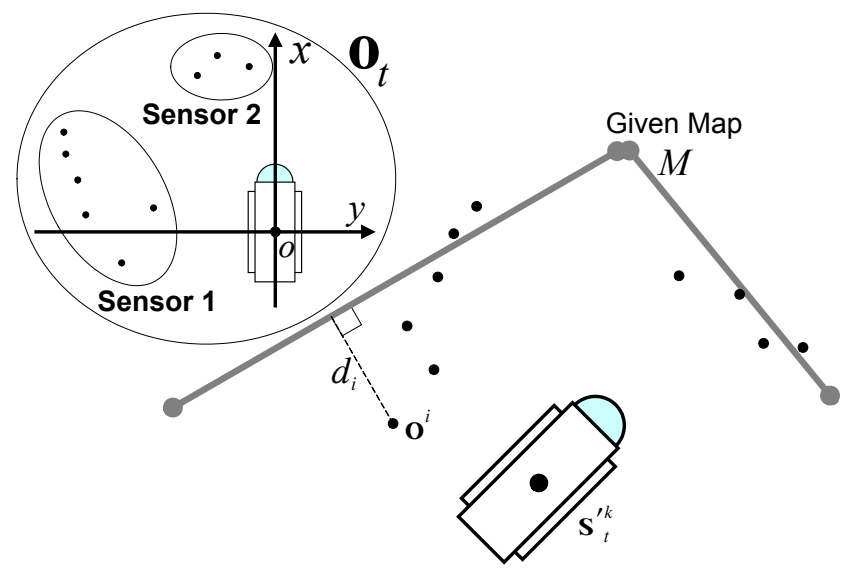

Fig. 3. Observation phase.

sample $\mathbf{S}_{t-1}^{i}$ is moved individually to $\mathbf{S}_{t}^{\prime i}$ including random noise of Gaussian distribution. The samples diffuse in time and this diffusion represents dead reckoning error. The standard deviations are estimated considering to the sensor specifications.

\subsubsection{Observation Phase}

The set $S_{t}$ is obtained from $S^{\prime}{ }_{t}$ by referring to the observation $O_{t}$, which is a set of positions of detected objects in the vehicle coordinates as shown in Fig. 3.

Each sample $\mathbf{s}_{t}^{\prime}{ }_{t}$ of the set $S^{\prime}{ }_{t}$ is weighted and resampled so that they are distributed in proportion to the likelihood $L\left(O_{t} \mid \mathbf{s}_{t}^{\prime i}, M\right)$. The re-sampled set results in $S_{t}$ that indicates the probability distribution function $p\left(\mathbf{x}_{t} \mid \mathbf{x}_{t-1}, \mathbf{a}_{t-1}, O_{t}, M\right)$.

Assuming that the observation $O$ consists of $n$ points $\mathbf{o}^{1}, \mathbf{o}^{2}, \cdots, \mathbf{o}^{n}$,

$$
L(O)=L\left(\mathbf{o}^{1}\right) L\left(\mathbf{o}^{2}\right) \cdots L\left(\mathbf{o}^{n}\right)
$$

is derived for each point is regarded as independent. As sensor error can be described as Gaussian distributions in general, the likelihood of the point $\mathbf{o}^{i}$ can be determined as follows using the shortest distance $d_{i}$ between $\mathbf{o}^{i}$ and the map $M$ as shown in Fig. 3:

$$
L\left(\mathbf{o}^{i} \mid \mathbf{s}^{\prime}{ }_{t}, M\right)=\mathrm{f}\left(d_{i}, \sigma\right)
$$

where $\mathrm{f}(x, \sigma)$ is distribution function:

$$
\mathrm{f}(x, \sigma)=\frac{1}{\sqrt{2 \pi} \sigma} \exp \left(\frac{-x^{2}}{2 \sigma^{2}}\right)
$$

There are concerns about serious error that result in a non-Gaussian distribution. For example, floating particles, debris and underwater creatures may disturb sensor measurements. Acoustic noise such as multipath, as well as large angle of incident, interfere measurement by sonar. To treat these errors correctly, the error coefficient $e$ is introduced and Eq.5 is applied only when all of the following conditions were satisfied, 


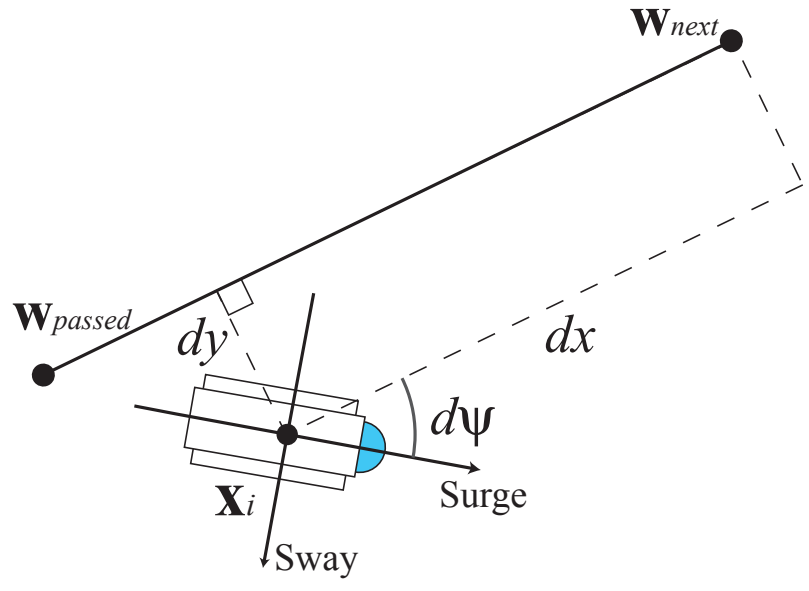

Fig. 4. Guidance of an AUV.

1) The segments of the map $M$ must be measured from correct side.

2) There must be no segments between the measured point and the sensor.

3) The shortest distance $d_{i}$ must be explained by a Gaussian, i.e. $d_{i}<e \sigma$.

4) The incident angle must be less than certain threshold (for sonar).

Otherwise,

$$
L\left(\mathbf{o}^{i} \mid \mathbf{s}^{\prime}{ }_{t}, M\right)=\mathrm{f}(e \sigma, \sigma)
$$

is applied. $\sigma$ is determined considering sensor accuracy $\sigma_{s}$ and uncertainty of the map $\sigma_{m}$.

\subsection{Guidance System}

Guidance System generates control reference to follow the observation path. When the sample set $S_{t}$ is well concentrated, the estimated $\mathbf{x}_{t}$ is regarded as an actual state and the Guidance System orders the vehicle to move. The aim of guidance is to zero the error $d x, d y$ and $d \psi$ shown in Fig. 4. The AUV surfaces up passing all the waypoints. The detail is reported in [6].

\section{IMPLEMENTATION}

The proposed method is implemented in the testbed AUV "Tri-Dog 1," which is approximately $1.8 \mathrm{~m}$ long and $170 \mathrm{~kg}$ in air. Six thrusters independently control surge, sway, heave and yaw motion. Sensors for the proposed method are described as below.

\subsection{Motion Sensors}

A Fiber Optic Gyro (FOG) measures heading rate and angle, and a Doppler Velocity Log (DVL) reads ground

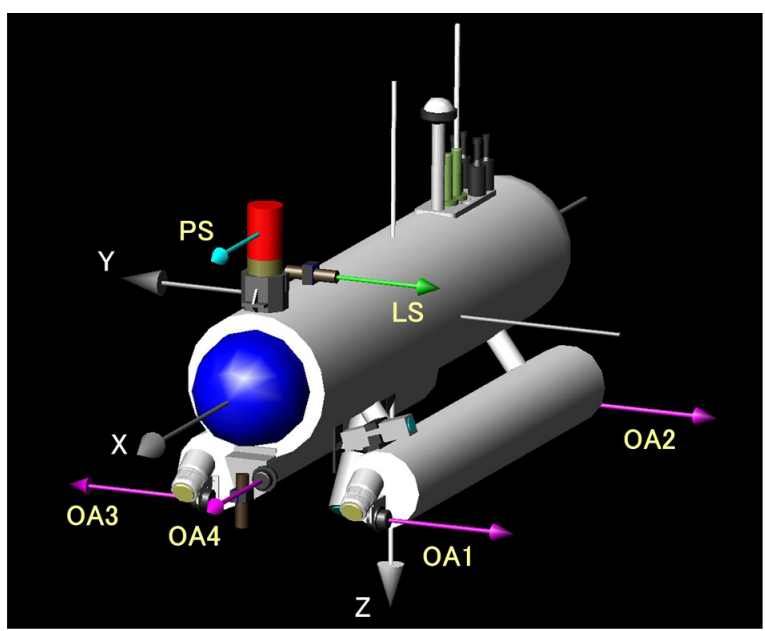

Fig. 5. Arrangement of perception sensors.

speed. These sensors are used to calculate the action $\mathbf{a}$. The vehicle also has an Attitude Heading Reference System (AHRS) and a depth meter.

\subsection{Perception Sensors}

The vehicle has four obstacle avoidance sonars (OA1 to OA4), one profiling sonar (PS) and one light-section profiler (LS) to acquire observation $O$, as shown in Fig. 5. Directivity of the obstacle avoidance sonars is about $20 \mathrm{deg}$ and its resolution is $0.01 \mathrm{~m}$. Coverage is set to be $5 \mathrm{~m}$ for the experiment. The profiling sonar has directivity of about 2 deg with a resolution of $0.01 \mathrm{~m}$. It mechanically scans $360 \mathrm{deg}$ on a horizontal plane with a step size of $2.4 \mathrm{deg}$. Coverage is set to be $40 \mathrm{~m}$ and rotation period is about $17 \mathrm{sec}$. The light-section profiler consists of a TV camera and a sheet laser device, providing continuous shape of target structures by light-sectioning. The authors proposed this system for AUV navigation and bathymetry mapping, and the performance has been verified though tank trials [10]. The resolution within a $2 \mathrm{~m}$ range is of millimeter order. It can provide simultaneously range data on a horizontal plane within $40 \mathrm{deg}$ at once. Coverage is set to be $3 \mathrm{~m}$.

\subsection{Observation Instruments}

There are three TV cameras (Cam1, Cam2, Cam3) onboard. Cam1 is mounted on AUV's nose directing forward, Cam2 is on its left. Cam3 is mounted on its bottom looking forward with $55 \mathrm{deg}$ depression. As well as being an observation tool Cam2 is also a component of LS and to enhance its performance it is mounted with $20 \mathrm{deg}$ elevation. There is another sheet laser device on AUV's nose, looking downward with the laser plane orthogonal to surge direction for bathymetry mapping. Its footprints are extracted from images of Cam3 by post processing. 


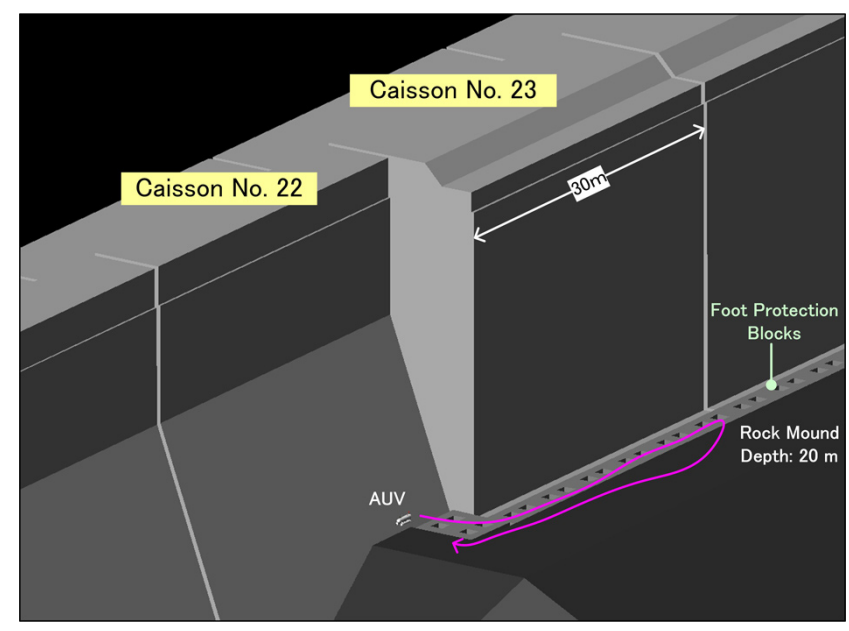

Fig. 6. Configuration of the breakwaters.

\section{EXPERIMENTS}

Experiments were carried out at the breakwaters of Kamaishi Bay, Iwate Prefecture in Japan. The experimental zone is shown in Fig. 6. Concrete caissons have a length of $30 \mathrm{~m}$ and are mounted on the rock mound. The depth of rock mound is about $20 \mathrm{~m}$ and there are foot protection blocks with a horizontal face $5 \mathrm{~m} \times 2.5 \mathrm{~m}$ laid along with the caissons.

\subsection{Experimental Mission}

Considering the practical application, two missions were planned. The observation path (waypoints) of each mission is shown in Figs. 7 and 8 by the circles. The environmental map $M$ is given as the configuration of the caissons' surface.

Mission 1 is a bottom survey. The vehicle observes the foot protection blocks and rock mound, maintaining a constant depth of $17.5 \mathrm{~m}$. The entire length of the path is about $211 \mathrm{~m}$. Mosaic and bathymetry map are made from images of Cam 3 by post processing.

Mission 2 is a wall survey. The vehicle observes the surface of caisson No. 23. The vehicle takes images of the surface by Cam2, traveling along with runs at multiple depth $(18 \mathrm{~m}, 15 \mathrm{~m}, 12 \mathrm{~m}, 9 \mathrm{~m})$. The entire length of the path is about $298 \mathrm{~m}$.

\subsection{Procedure}

The vehicle starts descending after being guided to the start area by an operator via wireless LAN. Upon reaching a depth of $2 \mathrm{~m}$, it initializes the particle filter and waits for the samples to concentrate. It then starts to track the observation path. The entire mission is carried out in full autonomy and there is no underwater communication. The vehicle surfaces and exits from autonomous mode after passing all the waypoints or if the vehicle decides it is impossible to continue the mission.

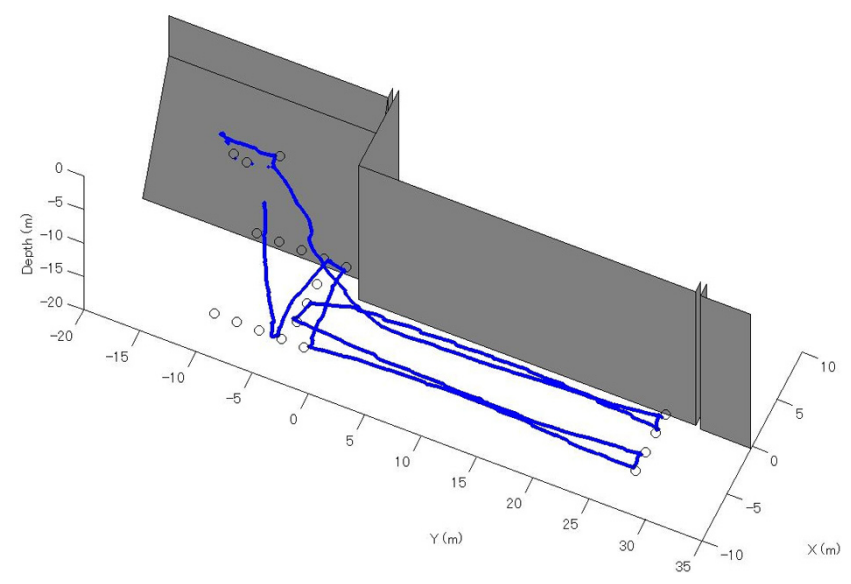

Fig. 7 Way points and the estimated trajectory of the Mission 1.

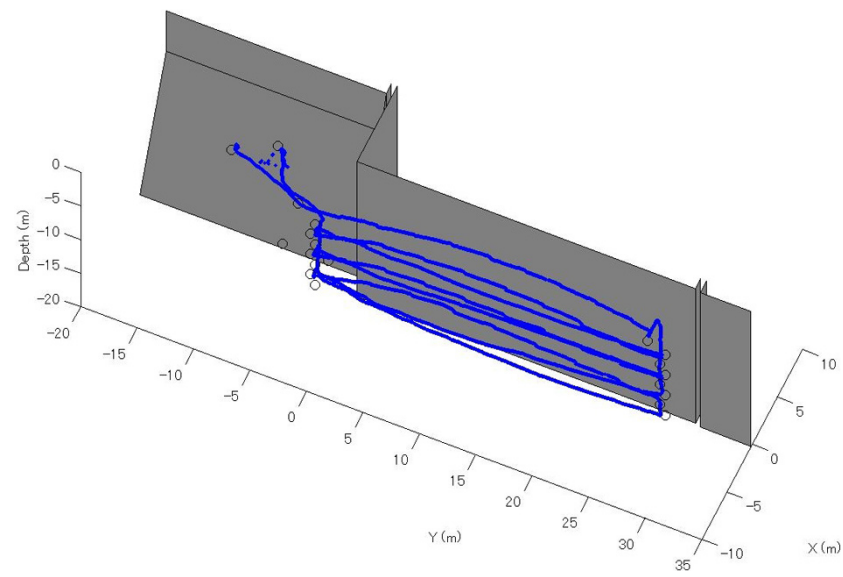

Fig. 8 Way points and the estimated trajectory of the Mission 2.

The number of samples $N$ of the particle filter is 500 with the update frequency $5 \mathrm{~Hz}$. The surge velocity is set to be $0.2 \mathrm{~m} / \mathrm{s}$.

\subsection{Results}

The vehicle succeeded in fully autonomous operation of $1049 \mathrm{sec}$, with a distance traveled of $166 \mathrm{~m}$ although it terminated the mission just after passing 12th waypoint because of low altitude (less than $0.5 \mathrm{~m}$ ) in the Mission 1 . Estimated trajectory is shown in Fig. 7. The mosaic of the bottom is shown in Fig. 9 with a $5 \mathrm{~m} \times 5 \mathrm{~m}$ grid superimposed. The distributions, shape and color of each rock and foot protection block can be observed. The mosaic is made considering not pictorial correlation but the estimated position and attitude of the vehicle. It follows that the estimated state has enough quality for practical application.

In the Mission 2, the vehicle passed the final 20th waypoint in $1423 \mathrm{sec}$ and completed the entire mission. Estimated trajectory is shown in Fig. 8. The mosaic of the caisson's surface was made with the images of Cam 2 as shown in Fig. 10. This mosaic was also made in the same 


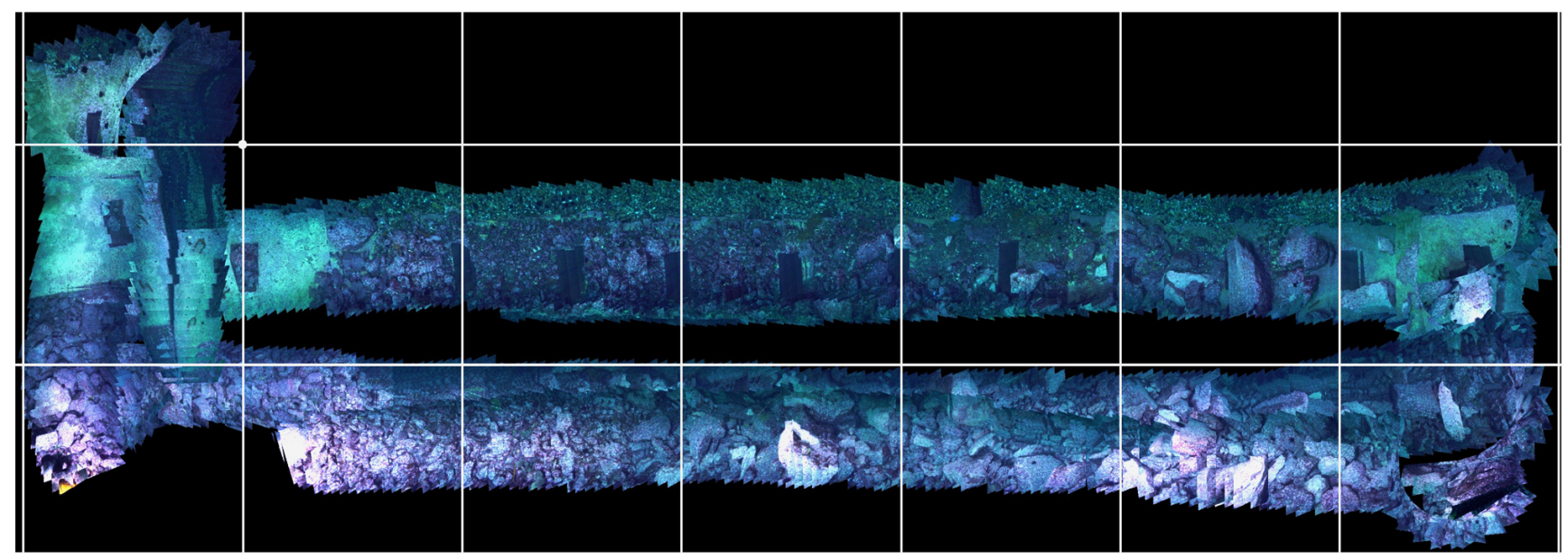

Fig. 9. Mosaic image of foot protection blocks and the rock mound.

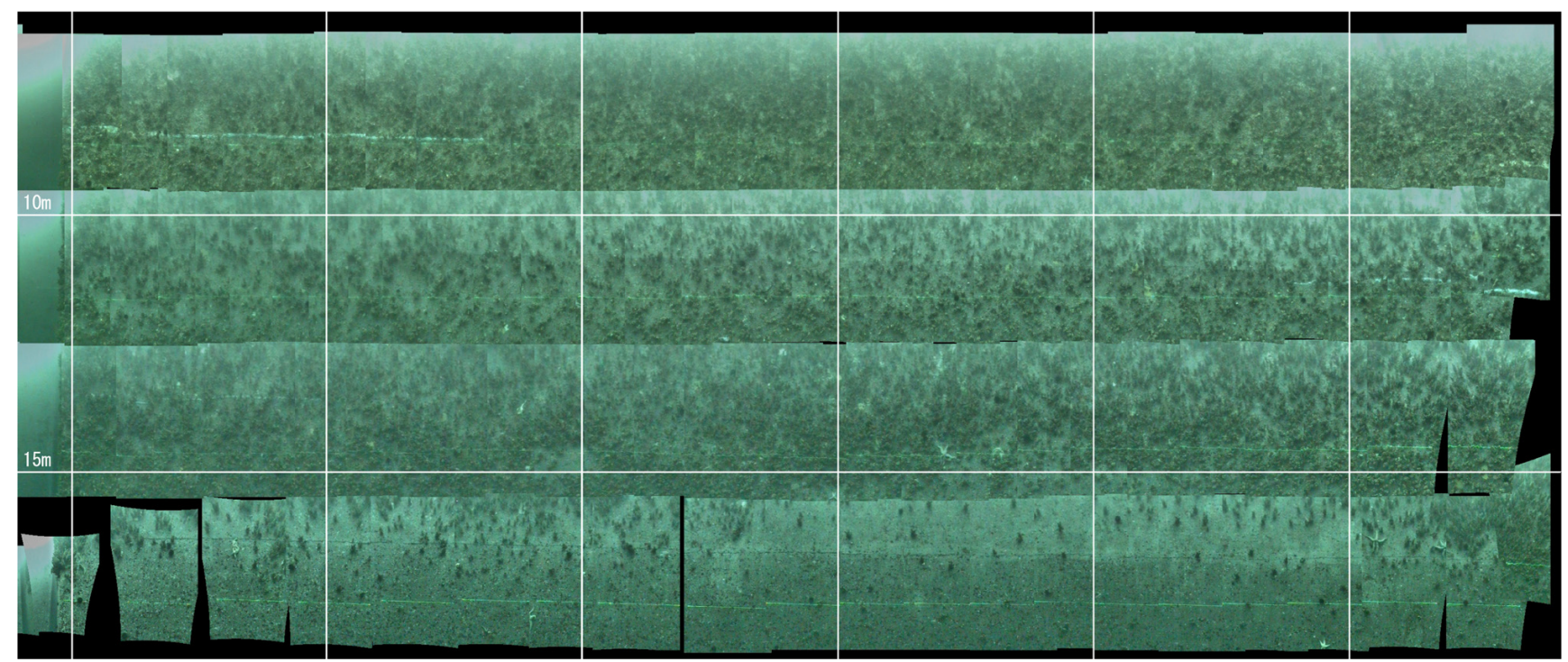

Fig. 10. Mosaic image of the caisson's surface.

way for Fig. 9. The distribution of biofoulings and surface condition can be seen over the entire wall.

\section{CONCLUSIONS}

This paper proposed a fusion based navigation method for an AUV operating around artificial structures. It realizes independent and robust navigation with environmental map and waypoints given. The method was implemented to the AUV "Tri-Dog 1" and experiments were carried out around breakwaters of Kamaishi Bay in Japan. The AUV succeeded in fully autonomous, independent operation, taking detailed images of the bottom and the caisson's surface. Mosaics of bottom features and caisson's surface were made over the entire covered area forming a detailed map of them. Thus, it can be concluded that this method is practically available.
The proposed method has also been modified and used for scientific observations of underwater vent areas [11, $12]$.

\section{ACKNOWLEDGMENTS}

This project is supported by The Service Center of Port Engineering (SCOPE) and Japan Society for the Promotion of Science (JSPS). The experiments were done as a part of joint project with The Port and Airport Research Institute (PARI). The authors would like to thank Kamaishi Port Construction Office -Tohoku Regional Development Bureau -Ministry of Land, Infrastructure and Transport, TOA CORPORATION, Kamaishi Ocean Development Ltd., and International Coastal Research Center -Ocean Research Institute -The University of Tokyo for providing us an opportunity and support of the sea trials. The authors 
would also like to thank Dr. Arjuna Balasuriya and Mr. Bharath Kalyan of Nanyang Technological University for their cooperation.

\section{REFERENCES}

[1] T. Asai, J. Kojima, K. Asakawa and T. Iso, "Inspection of submarine cable of over $400 \mathrm{~km}$ by AUV," Proc. of UT ’00, pp.133-135, 2000.

[2] H. Kondo and T. Ura, "Detailed object observation by autonomous underwater vehicle with localization involving uncertainty of magnetic bearings" Proc. of ICRA 2002, pp.412-419, 2002.

[3] P.E. An, A.J. Healey, J. Park and S.M.Smith, "Asynchronous Data Fusion For AUV Navigation Via Heuristic Fuzzy Filtering Techniques," Proc. of Oceans '97, pp.397-402, 1997.

[4] S. Majumder, S. Scheding and H.F. Durrant-Whyte, "Multisensor data fusion for underwater navigation," Robotics and Autonomous Systems, Vol.35, pp.97108, 2001.

[5] B. Kalyan, A. Balasuriya, "Multiple Sensors based Navigation Scheme for AUV Position Estimation," Proc. of UT '04, pp.201-207, 2004.

[6] H. Kondo, T. Maki, T. Ura, T. Sakamaki and M. Inaishi, "Relative Navigation of an AUV Using Image-and-
Acoustic Based Profiling System," Proc. of Oceans '04, pp.1330-1335, 2004.

[7] H. Kondo, T. Ura, Y. Nose, "Development of an Autonomous Underwater Vehicle "Tri-Dog" toward Practical Use in Shallow Water," Journal of Robotics and Mechatronics, Vol.13, No.2, pp.205-211, 2001.

[8] F. Dellaert, D. Fox, W. Burgard, S. Thrun, "Monte Carlo Localization for Mobile Robots," Proc. of ICRA 1999, pp.1322-1328, 1999.

[9] M. Adams, Sen Zhang and Lihua Xie, "Particle Filter based outdoor robot localization using natural features extracted from laser scanners," Proc. of ICRA '04, pp.1493-1498, 2004.

[10] H. Kondo, T. Maki, T. Ura, Y. Nose, T. Sakamaki and M. Inaishi, "Relative Navigation of an Autonomous Underwater Vehicle Using a Light-Section Profiling System," Proc. of IROS '04, pp.1103-1108, 2004.

[11] T. Maki, H. Kondo, T. Ura, T. Sakamaki, "Navigation of an Autonomous Underwater Vehicle for Photo Mosaicing of Shallow Vent Areas," Proc. of Oceans '06 Singapore, CD-ROM, 2006.

[12] T. Maki, H. Kondo, T. Ura, T. Sakamaki, "Photo Mosaicing of "Tagiri" Shallow Vent Area by the AUV "Tri-Dog 1" using a SLAM based Navigation Scheme," Proc. of Oceans '06 Boston (to be appeared in September, 2006). 